Article

\title{
A coupled fixed point theorem for maps satisfying rational type contractive condition in dislocated quasi b-metric space
}

\author{
Mesaud Tesfaye $^{1}$, Kidane Koyas ${ }^{1}$ and Solomon Gebregiorgis ${ }^{1, *}$ \\ 1 Department of Mathematics, Jimma University, Jimma, Ethiopia. \\ * Correspondence: solomonggty@gmail.com
}

Received: 20 August 2019; Accepted: 27 January 2020; Published: 7 March 2020.

\begin{abstract}
In this paper, a coupled fixed point theorem for maps satisfying rational type contractive condition in the perspective of dislocated quasi b-metric space have been formed and the existence and uniqueness of a coupled fixed point have been proved. Our result improves and generalizes comparable results in the literature.
\end{abstract}

Keywords: Continuous map, contractive condition, coupled fixed point, dislocated quasi $b$-metric space.

MSC: 47H06, 47H10, 54H25, 65D15.

\section{Introduction}

I $\mathrm{n}$ a complete dislocated metric space the celebrated Banach contraction principle was introduced and generalized by [1,2]. Since then, Zeyada [3] introduced the notion of dislocated quasi metric space for the first time. In this space, self-distance need not to be necessarily zero which is a very remarkable property of this space. After that the idea of dislocated quasi b-metric space was presented in [4].

The concept of coupled fixed point was extended by Guo \& Lakshmikantham [5], where the monotone iterations technique is exploited. After that they introduced the concept for Partially ordered set. Bhaskar \& Lakshmikantham [6] studied the existence and uniqueness of a coupled fixed point results in partially ordered metric space. They also introduced the concept of coupled fixed point and proved some fixed point theorems under certain contractive conditions. Moreover, after the work of Bhaskar \& Lakshmikantham [6] coupled fixed point results were studied by many authors in different type of spaces [7]. Recently, Mohammed et al. [8] proved a coupled fixed point result in the setting of dislocated quasi-metric spaces.

Provoked by the result of Mohammed et al. [8], in this paper, a coupled fixed point theorem for maps satisfying rational type contractive condition in the perspective of dislocated quasi b-metric spaces have been established. Our result extends, improves and generalizes the comparable results in the existing literature. Moreover, we provided an example to support our main result.

\section{Preliminaries}

Throughout this paper, $R^{+}$represents the set of non-negative real numbers and $N$ represents the set of natural numbers. First, we recall some known definitions and lemmas.

Definition 1. [4] Let $X \neq \varnothing$ and $d: X \times X \rightarrow[0, \infty)$ be a function satisfying the conditions:

1. $d(x, y)=d(y, x)=0 \Rightarrow x=y$,

2. $d(x, y) \leq k[d(x, z)+d(z, y)]$ for all $x, y, z \in X$.

Where $k \geq 1$ is any given real number. Then $d$ is known as dislocated quasi b-metric on $X$ and the pair $(X, d)$ is called a dislocated quasi $b$-metric space or in short $(\mathrm{dqb})$ metric spaces.

Definition 2. [4] A sequence $\left\{x_{n}\right\}$ in a dislocated quasi b-metric space $(X, d)$ is said to converge to a point $x \in X$ if and only if

$$
\lim _{n \rightarrow \infty} d\left(x_{n}, x\right)=0=\lim _{n \rightarrow \infty} d\left(x, x_{n}\right) .
$$


Definition 3. [4] A sequence $\left\{x_{n}\right\}$ in a dq b-metric space $(X, d)$ is said be a Cauchy sequence if for each $\epsilon>0$, there exists a positive integer $n_{0}$ such that for all $n, m \geq n_{0}$, we have $d\left(x_{n}, x_{m}\right)<\epsilon$. That is, $\lim _{n, m \rightarrow \infty} d\left(x_{n}, x_{m}\right)=0$.

Definition 4. [4] A dislocated quasi b-metric space is called complete if every Cauchy sequence converges to an element in the same metric space.

Definition 5. [4] Let $\left(X, d_{1}\right)$ and $\left(Y, d_{2}\right)$ be two dislocated quasi b-metric spaces, then the mapping $T: X \rightarrow Y$ is said to be continuous if for each sequence $\left\{x_{n}\right\}$ which is convergent to $x_{0}$ in $X$, the sequence $\left\{T x_{n}\right\}$ converges to $T x_{0}$ in $Y$.

Lemma 1. [4] In dislocated quasi b-metric space, the limit of a convergent sequence is unique.

Definition 6. [9] Let $T: X \rightarrow X$ be a self-map in a complete metric space $(X, d)$. Then $T$ is said to be a contraction mapping if there exist a constant $k \in[0,1)$ such that

$$
d(T x, T y) \leq k d(x, y) \quad \text { for all } x, y \in X .
$$

Definition 7. Let $X$ be a nonempty set and $T: X \rightarrow X$ be a self-map. We say that $x$ is a fixed point of $T$ if $T x=x$.

Theorem 1. [10] Let $T: X \rightarrow X$ be a continuous contraction with $\lambda \in[0,1)$ and $0 \leq \lambda<\frac{1}{k}$ for $k \geq 1$ in a complete dislocated quasi-b-metric space $(X, d)$, then $T$ has a unique fixed point in $X$.

Definition 8. [6] Let $X$ be any non-empty set. An element $(x, y) \in X \times X$ is said to be a coupled fixed point of the mapping $T: X \rightarrow X$ if $T(x, y)=x$ and $T(y, x)=y$.

Theorem 2. [8] Let $(X, d)$ be a complete dislocated quasi-metric space and $T: X \rightarrow X$ be a continuous mapping satisfying the following rational type contractive conditions

$$
\begin{aligned}
d[T(x, y), T(u, v)] \leq & a_{1}[d(x, u)+d(y, v)]+a_{2}[d(x, T(x, y))+d(u, T(u, v))] \\
& +a_{3}[d(x, T(u, v))+d(u, T(x, y))]+a_{4}\left[\frac{d(x, T(x, y)) d(u, T(u, v))}{d(x, u)+d(y, v)}\right] \\
& +a_{5}\left[\frac{[d(x, u)+d(y, v)]}{[d(x, T(x, y))+d(u, T(u, v))]}[1+d(x, u)+d(y, v)]\right] \\
& +a_{6}\left[\frac{d(x, T(x, y))+d(x, T(u, v))}{1+d(u, T(u, v)) d(u, T(x, y))}\right]
\end{aligned}
$$

for all $x, y, u, v \in X$ and all $a_{1}, a_{2}, a_{3}, a_{4}, a_{5}, a_{6}$ are non-negative constants with $2\left(a_{1}+a_{2}+a_{5}\right)+4\left(a_{3}+a_{6}\right)+a_{4}<1$. Then $T$ has a unique coupled fixed point in $X \times X$.

\section{Main Results}

Theorem 3. Let $(X, d)$ be a complete dislocated quasi b-metric space with constant coefficient $k \geq 1$ and $T: X \times X \rightarrow X$ be a continuous map satisfying the following rational type contractive conditions:

$$
\begin{aligned}
d[T(x, y), T(u, v)] \leq & a_{1}[d(x, u)+d(y, v)]+a_{2}[d(x, T(x, y))+d(u, T(u, v))]+a_{3}[d(x, T(u, v))+d(u, T(x, y))] \\
& +a_{4}\left[\frac{d(x, T(x, y)) d(u, T(u, v))}{d(x, u)+d(y, v)}\right]+a_{5}\left[\frac{[d(x, u)+d(y, v)]}{[d(x, T(x, y))+d(u, T(u, v))]}[1+d(x, u)+d(y, v)]\right] \\
& +a_{6}\left[\frac{d(u, T(x, y))+d(x, T(u, v))}{1+d(u, T(u, v)) d(u, T(x, y))}\right]+a_{7}\left[\frac{[d(y, v)+d(x, T(x, y))] d(u, T(u, v))}{1+d(y, v)+d(x, T(x, y))}\right]
\end{aligned}
$$

for all $x, y, u, v \in X$ and $a_{1}, a_{2}, a_{3}, a_{4}, a_{5}, a_{6}$, and $a_{7}$ are non-negative constants with $2 k a_{1}+(k+1)\left(a_{2}+a_{5}\right)+\left(2 k^{2}+\right.$ $2 k)\left(a_{3}+a_{6}\right)+a_{4}+a_{7}<1$. Then $T$ has a unique coupled fixed point in $X \times X$.

Proof. Let $x_{0}, y_{0} \in X$ be any two arbitrary points. We can construct two sequences $\left\{x_{n}\right\}$ and $\left\{y_{n}\right\} \in$ $X$ such that $x_{n+1}=T\left(x_{n}, y_{n}\right)$ and $y_{n+1}=T\left(y_{n}, x_{n}\right)$ for $n=0,1,2, \cdots$. Consider, $d\left(x_{n}, x_{n+1}\right)=$ $d\left[T\left(x_{n-1}, y_{n-1}\right), T\left(x_{n}, y_{n}\right)\right]$. 
Now, we have:

$$
\begin{aligned}
d\left(x_{n}, x_{n+1}\right)= & d\left[T\left(x_{n-1}, y_{n-1}\right), T\left(x_{n}, y_{n}\right)\right] \\
\leq & a_{1}\left[d\left(x_{n-1}, x_{n}\right)+d\left(y_{n-1}, y_{n}\right)\right]+a_{2}\left[d\left(x_{n-1}, T\left(x_{n-1}, y_{n-1}\right)\right)+d\left(x_{n}, T\left(x_{n}, y_{n}\right)\right)\right] \\
& +a_{3}\left[d\left(x_{n-1}, T\left(x_{n}, y_{n}\right)\right)+d\left(x_{n}, T\left(x_{n-1}, y_{n-1}\right)\right)\right]+a_{4}\left[\frac{d\left(x_{n-1}, T\left(x_{n-1}, y_{n-1}\right)\right) d\left(x_{n}, T\left(x_{n}, y_{n}\right)\right)}{d\left(x_{n-1}, x_{n}\right)+d\left(y_{n-1}, y_{n}\right)}\right] \\
& +a_{5}\left[\frac{\left[d\left(x_{n-1}, x_{n}\right)+d\left(y_{n-1}, y_{n}\right)\right]}{\left[d\left(x_{n-1}, T\left(x_{n-1}, y_{n-1}\right)\right)+d\left(x_{n}, T\left(x_{n}, y_{n}\right)\right)\right]}\left[1+d\left(x_{n-1}, x_{n}\right)+d\left(y_{n-1}, y_{n}\right)\right]\right] \\
& +a_{6}\left[\frac{d\left(x_{n}, T\left(x_{n-1}, y_{n-1}\right)\right)+d\left(x_{n-1}, T\left(x_{n}, y_{n}\right)\right)}{1+d\left(x_{n}, T\left(x_{n}, y_{n}\right)\right) d\left(x_{n}, T\left(x_{n-1}, y_{n-1}\right)\right)}\right] \\
& +a_{7}\left[\frac{\left[d\left(y_{n-1}, y_{n}\right)+d\left(x_{n-1}, T\left(x_{n-1}, y_{n-1}\right)\right)\right] d\left(x_{n}, T\left(x_{n}, y_{n}\right)\right)}{1+d\left(y_{n-1}, y_{n}\right)+d\left(x_{n-1}, T\left(x_{n-1}, y_{n-1}\right)\right.}\right] .
\end{aligned}
$$

By using the definitions of the sequences $\left\{x_{n}\right\}$ and $\left\{y_{n}\right\}$, we have:

$$
\begin{aligned}
d\left(x_{n}, x_{n+1}\right) \leq & a_{1}\left[d\left(x_{n-1}, x_{n}\right)+d\left(y_{n-1}, y_{n}\right)\right]+a_{2}\left[d\left(x_{n-1}, x_{n}\right)+d\left(x_{n}, x_{n+1}\right)\right] \\
& +a_{3}\left[d\left(x_{n-1}, x_{n+1}\right)+d\left(x_{n}, x_{n}\right)\right]+a_{4}\left[\frac{d\left(x_{n-1}, x_{n}\right) d\left(x_{n}, x_{n+1}\right)}{d\left(x_{n-1}, x_{n}\right)+d\left(y_{n-1}, y_{n}\right)}\right] \\
& +a_{5}\left[\frac{\left[d\left(x_{n-1}, x_{n}\right)+d\left(y_{n-1}, y_{n}\right)\right]\left[d\left(x_{n-1}, x_{n}\right)+d\left(x_{n}, x_{n+1}\right)\right]}{\left.1+d\left(x_{n-1}, x_{n}\right)+\left(y_{n-1}, y_{n}\right)\right]}\right] \\
& +a_{6}\left[\frac{d\left(x_{n}, x_{n}\right)+d\left(x_{n-1}, x_{n+1}\right)}{1+d\left(x_{n}, x_{n+1}\right) d\left(x_{n}, x_{n}\right)}\right]+a_{7}\left[\frac{\left[d\left(y_{n-1}, y_{n}\right)+d\left(x_{n-1}, x_{n}\right)\right] d\left(x_{n}, x_{n+1}\right)}{1+d\left(y_{n-1}, y_{n}\right)+d\left(x_{n-1}, x_{n}\right)}\right] .
\end{aligned}
$$

Using the triangle inequality and the fact that $d(x, y) \geq 0$, we have:

$$
\begin{aligned}
d\left(x_{n}, x_{n+1}\right)= & d\left[T\left(x_{n-1}, y_{n-1}\right), T\left(x_{n}, y_{n}\right)\right] \\
\leq \quad & a_{1}\left[d\left(x_{n-1}, x_{n}\right)+d\left(y_{n-1}, y_{n}\right)\right] \\
& +a_{2}\left[d\left(x_{n-1}, x_{n}\right)+d\left(x_{n}, x_{n+1}\right)\right]+k a_{3}\left[d\left(x_{n-1}, x_{n}\right)+d\left(x_{n}, x_{n+1}\right)+d\left(x_{n-1}, x_{n}\right)+d\left(x_{n}, x_{n+1}\right)\right] \\
& +a_{4}\left[\frac{d\left(x_{n-1}, x_{n}\right) d\left(x_{n}, x_{n+1}\right)}{d\left(x_{n-1}, x_{n}\right)+d\left(y_{n-1}, y_{n}\right)}\right]+a_{5}\left[\frac{\left[d\left(x_{n-1}, x_{n}\right)+d\left(y_{n-1}, y_{n}\right)\right]\left[d\left(x_{n-1}, x_{n}\right)+d\left(x_{n}, x_{n+1}\right)\right]}{1+d\left(x_{n-1}, x_{n}\right)+d\left(y_{n-1}, y_{n}\right)}\right] \\
& +k a_{6}\left[\frac{d\left(x_{n-1}, x_{n}\right)+d\left(x_{n}, x_{n+1}\right)+d\left(x_{n-1}, x_{n}\right)+d\left(x_{n}, x_{n+1}\right)}{1+d\left(x_{n}, x_{n+1}\right) d\left(x_{n}, x_{n+1}\right)}\right] \\
& +a_{7}\left[\frac{\left[d\left(y_{n-1}, y_{n}\right)+d\left(x_{n-1}, x_{n}\right)\right] d\left(x_{n}, x_{n+1}\right)}{1+d\left(y_{n-1}, y_{n}\right)+d\left(x_{n-1}, x_{n}\right)}\right] .
\end{aligned}
$$

Simplifying the above inequality, we have:

$$
\begin{aligned}
d\left(x_{n}, x_{n+1}\right) \leq & a_{1}\left[d\left(x_{n-1}, x_{n}\right)+d\left(y_{n-1}, y_{n}\right)\right]+a_{2}\left[d\left(x_{n-1}, x_{n}\right)+d\left(x_{n}, x_{n+1}\right)\right] \\
& +k a_{3}\left[d\left(x_{n-1}, x_{n}\right)+d\left(x_{n}, x_{n+1}\right)+d\left(x_{n-1}, x_{n}\right)+d\left(x_{n}, x_{n+1}\right)\right] \\
& +a_{4} d\left(x_{n}, x_{n+1}\right)+a_{5}\left[d\left(x_{n-1}, x_{n}\right)+d\left(x_{n}, x_{n+1}\right)\right]+k a_{6}\left[d\left(x_{n-1}, x_{n}\right)\right. \\
& \left.+d\left(x_{n}, x_{n+1}\right)+d\left(x_{n-1}, x_{n}\right)+d\left(x_{n}, x_{n+1}\right)\right]+a_{7} d\left(x_{n}, x_{n+1}\right) .
\end{aligned}
$$

It follows that:

$$
\begin{aligned}
d\left(x_{n}, x_{n+1}\right) \leq & \left(a_{2}+2 k\left(a_{3}+a_{6}\right)+a_{4}+a_{5}+a_{7}\right) d\left(x_{n}, x_{n+1}\right) \\
& +\left(a_{1}+a_{2}+2 k\left(a_{3}+a_{6}\right)+a_{5}\right) d\left(x_{n-1}, x_{n}\right)+a_{1} d\left(y_{n-1}, y_{n}\right) .
\end{aligned}
$$

Simplification yields:

$$
\begin{aligned}
d\left(x_{n}, x_{n+1}\right) \leq & \frac{a_{1}+a_{2}+2 k\left(a_{3}+a_{6}\right)+a_{5}}{1-\left(a_{2}+2 k\left(a_{3}+a_{6}\right)+a_{4}+a_{5}+a_{7}\right)} d\left(x_{n-1}, x_{n}\right) \\
& +\frac{a_{1}}{1-\left(a_{2}+2 k\left(a_{3}+a_{6}\right)+a_{4}+a_{5}+a_{7}\right)} d\left(y_{n-1}, y_{n}\right) .
\end{aligned}
$$


Proceeding similarly, we can show that:

$$
\begin{aligned}
d\left(y_{n}, y_{n+1}\right) \leq & \frac{a_{1}+a_{2}+2 k\left(a_{3}+a_{6}\right)+a_{5}}{1-\left(a_{2}+2 k\left(a_{3}+a_{6}\right)+a_{4}+a_{5}+a_{7}\right)} d\left(y_{n-1}, y_{n}\right) \\
& +\frac{a_{1}}{1-\left(a_{2}+2 k\left(a_{3}+a_{6}\right)+a_{4}+a_{5}+a_{7}\right)} d\left(x_{n-1}, x_{n}\right)
\end{aligned}
$$

Adding (1) and (2), we get:

$$
\left[d\left(x_{n}, x_{n+1}\right)+d\left(y_{n}, y_{n+1}\right)\right] \leq \frac{2 a_{1}+a_{2}+2 k\left(a_{3}+a_{6}\right)+a_{5}}{1-\left(a_{2}+2 k\left(a_{3}+a_{6}\right)+a_{4}+a_{5}+a_{7}\right)}\left[d\left(x_{n-1}, x_{n}\right)+d\left(y_{n-1}, y_{n}\right)\right]
$$

Since $2 k a_{1}+(k+1)\left(a_{2}+a_{5}\right)+\left(2 k^{2}+2 k\right)\left(a_{3}+a_{6}\right)+a_{4}+a_{7}<1$ with

$$
\lambda=\frac{2 a_{1}+a_{2}+2 k\left(a_{3}+a_{6}\right)+a_{5}}{1-\left(a_{2}+2 k\left(a_{3}+a_{6}\right)+a_{4}+a_{5}+a_{7}\right)}<1 .
$$

So, we have $k \lambda<1$, thus, the above inequality becomes:

$$
\left[d\left(x_{n}, x_{n+1}\right)+d\left(y_{n}, y_{n+1}\right)\right] \leq \lambda\left[d\left(x_{n-1}, x_{n}\right)+d\left(y_{n-1}, y_{n}\right)\right]
$$

Furthermore,

$$
\begin{aligned}
d\left(x_{n+1}, x_{n+2}\right) \leq & \frac{a_{1}+a_{2}+2 k\left(a_{3}+a_{6}\right)+a_{5}}{1-\left(a_{2}+2 k\left(a_{3}+a_{6}\right)+a_{4}+a_{5}+a_{7}\right)} d\left(x_{n}, x_{n+1}\right) \\
& +\frac{a_{1}}{1-\left(a_{2}+2 k\left(a_{3}+a_{6}\right)+a_{4}+a_{5}+a_{7}\right)} d\left(y_{n}, y_{n+1}\right)
\end{aligned}
$$

Similarly,

$$
\begin{aligned}
d\left(y_{n+1}, y_{n+2}\right) \leq & \frac{a_{1}+a_{2}+2 k\left(a_{3}+a_{6}\right)+a_{5}}{1-\left(a_{2}+2 k\left(a_{3}+a_{6}\right)+a_{4}+a_{5}+a_{7}\right)} d\left(y_{n}, y_{n+1}\right) \\
& +\frac{a_{1}}{1-\left(a_{2}+2 k\left(a_{3}+a_{6}\right)+a_{4}+a_{5}+a_{7}\right)} d\left(x_{n}, x_{n+1}\right)
\end{aligned}
$$

Adding (3) and (4), we obtain:

$$
\begin{aligned}
{\left[d\left(x_{n+1}, x_{n+2}\right)+d\left(y_{n+1}, y_{n+2}\right)\right] } & \leq \lambda\left[d\left(x_{n}, x_{n+1}\right)+d\left(y_{n}, y_{n+1}\right)\right] \\
& =\lambda^{2}\left[d\left(x_{n-1}, x_{n}\right)+d\left(y_{n-1}, y_{n}\right)\right]
\end{aligned}
$$

Continuing this process inductively, we have:

$$
\left[d\left(x_{n}, x_{n+1}\right)+d\left(y_{n}, y_{n+1}\right)\right] \leq \lambda^{n}\left[d\left(x_{0}, x_{1}\right)+d\left(y_{0}, y_{1}\right)\right] .
$$

Now, we show that $\left\{x_{n}\right\}$ and $\left\{y_{n}\right\}$ are Cauchy sequences in $X$. For non negative integers $m$ and $n$ with $m>n$, we have

$$
\begin{aligned}
{\left[d\left(x_{n}, x_{m}\right)+d\left(y_{n}, y_{m}\right)\right] \leq } & k\left[d\left(x_{n}, x_{n+1}\right)+d\left(x_{n+1}, x_{m}\right)+d\left(y_{n}, y_{n+1}\right)+d\left(y_{n+1}, y_{m}\right)\right] \\
\leq & k\left[d\left(x_{n}, x_{n+1}\right)+d\left(y_{n}, y_{n+1}\right)\right]+k^{2}\left[d\left(x_{n+1}, x_{n+2}\right)+\left(d\left(y_{n+1}, y_{n+2}\right)\right]\right. \\
& +\ldots+k^{m-n}\left[d\left(x_{m-1}, x_{m}\right)+d\left(y_{m-1}, y_{m}\right)\right] \\
\leq & k \lambda^{n}\left[d\left(x_{0}, x_{1}\right)+d\left(y_{0}, y_{1}\right)\right]+k^{2} \lambda^{n+1}\left[d\left(x_{0}, x_{1}\right)+d\left(y_{0}, y_{1}\right)\right] \\
& +\ldots+k^{m-n} \lambda^{m-1}\left[d\left(x_{0}, x_{1}\right)+d\left(y_{0}, y_{1}\right)\right] \\
= & k \lambda^{n}\left(1+k \lambda+(k \lambda)^{2}+\ldots+(k \lambda)^{m-n-1}\right)\left[d\left(x_{0}, x_{1}\right)+d\left(y_{0}, y_{1}\right)\right] \\
\leq & \frac{k \lambda^{n}}{1-k \lambda}\left[d\left(x_{0}, x_{1}\right)+d\left(y_{0}, y_{1}\right)\right] \rightarrow 0 \text { as } n \rightarrow \infty
\end{aligned}
$$

It follows that:

$$
\left[d\left(x_{n}, x_{m}\right)+d\left(y_{n}, y_{m}\right)\right] \rightarrow 0 \text { as } n, m \rightarrow \infty
$$


which in turn implies that:

$$
d\left(x_{n}, x_{m}\right) \rightarrow 0 \text { and } d\left(y_{n}, y_{m}\right) \rightarrow 0 \text { as } n, m \rightarrow \infty .
$$

Hence $\left\{x_{n}\right\}$ and $\left\{y_{n}\right\}$ are Cauchy sequences in a complete dislocated quasi b-metric space $X$. As a result there must exist $(x, y) \in X \times X$ such that $x_{n} \rightarrow x$ and $y_{n} \rightarrow y$ as $n \rightarrow \infty$. In addition, Since $T$ is continuous we have:

$$
x=\lim _{n \rightarrow \infty} x_{n+1}=\lim _{n \rightarrow \infty} T\left(x_{n}, y_{n}\right)=T\left(\lim _{n \rightarrow \infty} x_{n}, \lim _{n \rightarrow \infty} y_{n}\right)=T(x, y) .
$$

Similarly,

$$
y=\lim _{n \rightarrow \infty} y_{n+1}=\lim _{n \rightarrow \infty} T\left(y_{n}, x_{n}\right)=T\left(\lim _{n \rightarrow \infty} y_{n}, \lim _{n \rightarrow \infty} x_{n}\right)=T(y, x) .
$$

Therefore, $(x, y) \in X \times X$ is a coupled fixed point of $T$.

\section{Uniqueness}

Now, we show that $(x, y) \in X \times X$ is a unique coupled fixed point of $T$. Suppose that $T$ has another different coupled fixed point say $\left(x^{\prime}, y^{\prime}\right)$ where $\left(x^{\prime}, y^{\prime}\right) \in X \times X$ with $x^{\prime}=T\left(x^{\prime}, y^{\prime}\right)$ and $y^{\prime}=T\left(y^{\prime}, x^{\prime}\right)$. Then, by using (1), we have:

$$
\begin{aligned}
d(x, x)= & d[T(x, y), T(x, y)] \\
\leq & a_{1}[d(x, x)+d(y, y)]+a_{2}[d(x, T(x, y))+d(x, T(x, y))]+a_{3}[d(x, T(x, y))+d(x, T(x, y))] \\
& +a_{4}\left[\frac{d(x, T(x, y)) d(x, T(x, y))}{d(x, x)+d(y, y)}\right]+a_{5}\left[\frac{[d(x, x)+d(y, y)][d(x, T(x, y))+d(x, T(x, y))]}{1+d(x, x)+d(y, y)}\right] \\
& +a_{6}\left[\frac{d(x, T(x, y))+d(x, T(x, y))}{1+d(x, T(x, y)) d(x, T(x, y))}\right]+a_{7}\left[\frac{[d(y, y)+d(x, T(x, y))] d(x, T(x, y))}{1+d(y, y)+d(x, T(x, y))}\right] . \\
= & a_{1}[d(x, x)+d(y, y)]+a_{2}[d(x, x)+d(x, x)]+a_{3}[d(x, x)+d(x, x)]+a_{4}\left[\frac{d(x, x) d(x, x)}{d(x, x)+d(y, y)}\right] \\
& +a_{5}\left[\frac{[d(x, x)+d(y, y)][d(x, x)+d(x, x)]}{1+d(x, x)+d(y, y)}\right]+a_{6}\left[\frac{d(x, x)+d(x, x)}{1+d(x, x) d(x, x)}\right] \\
& +a_{7}\left[\frac{[d(y, y)+d(x, x)] d(x, x)}{1+d(y, y)+d(x, x)}\right] . \\
\leq & a_{1}[d(x, x)+d(y, y)]+a_{2}[d(x, x)+d(x, x)]+a_{3}[d(x, x)+d(x, x)]+a_{4} d(x, x) \\
& +a_{5}[d(x, x)+d(x, x)]+a_{6}[d(x, x)+d(x, x)]+a_{7} d(x, x) .
\end{aligned}
$$

Then simplification yields:

$$
d(x, x) \leq \delta d(x, x)+a_{1} d(y, y) .
$$

where $\delta=a_{1}+2\left(a_{2}+a_{3}+a_{5}+a_{6}\right)+a_{4}+a_{7}$.

In a similar way, we can confirm that:

$$
d(y, y) \leq \delta d(y, y)+a_{1} d(x, x) .
$$

Adding (6) and (7), we obtain:

$$
[d(x, x)+d(y, y)] \leq \mu[d(x, x)+d(y, y)] .
$$

where $\mu=\delta+a_{1}$.

Since, $\mu<1$ hence the above inequality is possible if and only if $d(x, x)+d(y, y)=0$. Hence $d(x, x)=0$ and $d(y, y)=0$. Similarly, $d\left(x^{\prime}, x^{\prime}\right)=0$ and $d\left(y^{\prime}, y^{\prime}\right)=0$. Now, we consider:

$$
\begin{aligned}
d\left(x, x^{\prime}\right)= & d\left[T(x, y), T\left(x^{\prime}, y^{\prime}\right)\right] \\
\leq & a_{1}\left[d\left(x, x^{\prime}\right)+d\left(y, y^{\prime}\right)\right]+a_{2}\left[d(x, T(x, y))+d\left(x^{\prime}, T\left(x^{\prime}, y^{\prime}\right)\right)\right] \\
& +a_{3}\left[d\left(x, T\left(x^{\prime}, y^{\prime}\right)\right)+d\left(x^{\prime}, T(x, y)\right)\right]+a_{4}\left[\frac{d(x, T(x, y)) d\left(x^{\prime}, T\left(x^{\prime}, y^{\prime}\right)\right)}{d\left(x, x^{\prime}\right)+d\left(y, y^{\prime}\right)}\right]
\end{aligned}
$$




$$
\begin{aligned}
& +a_{5}\left[\frac{\left[d\left(x, x^{\prime}\right)+d\left(y, y^{\prime}\right)\right]\left[d(x, T(x, y))+d\left(x^{\prime}, T\left(x^{\prime}, y^{\prime}\right)\right)\right]}{1+d\left(x, x^{\prime}\right)+d\left(y, y^{\prime}\right)}\right] \\
& +a_{6}\left[\frac{d\left(x^{\prime}, T(x, y)\right)+d\left(x, T\left(x^{\prime}, y^{\prime}\right)\right)}{1+d\left(x^{\prime}, T\left(x^{\prime}, y^{\prime}\right)\right) d\left(x^{\prime}, T(x, y)\right)}\right]+a_{7}\left[\frac{d\left(y, y^{\prime}\right)+d(x, T(x, y)) d\left(x^{\prime}, T\left(x^{\prime}, y^{\prime}\right)\right)}{1+d\left(y, y^{\prime}\right)+d\left(x^{\prime}, T\left(x^{\prime}, y^{\prime}\right)\right)}\right] .
\end{aligned}
$$

In fact $T(x, y)=x$ and $T\left(x^{\prime}, y^{\prime}\right)=x^{\prime}$, then we have:

$$
\begin{aligned}
d\left(x, x^{\prime}\right)= & a_{1}\left[d\left(x, x^{\prime}\right)+d\left(y, y^{\prime}\right)\right]+a_{2}\left[d(x, x)+d\left(x^{\prime}, x^{\prime}\right)\right]+a_{3}\left[d\left(x, x^{\prime}\right)+d\left(x^{\prime}, x\right)\right]+a_{4}\left[\frac{d(x, x) d\left(x^{\prime}, x^{\prime}\right)}{d\left(x, x^{\prime}\right)+d\left(y, y^{\prime}\right)}\right] \\
& +a_{5}\left[\frac{\left[d\left(x, x^{\prime}\right)+d\left(y, y^{\prime}\right)\right]\left[d(x, x)+d\left(x^{\prime}, x^{\prime}\right)\right]}{1+d\left(x, x^{\prime}\right)+d\left(y, y^{\prime}\right)}\right]+a_{6}\left[\frac{d\left(x^{\prime}, x\right)+d\left(x, x^{\prime}\right)}{1+d\left(x^{\prime}, x^{\prime}\right) d\left(x^{\prime}, x\right)}\right] \\
& +a_{7}\left[\frac{\left[d\left(y, y^{\prime}\right)+d\left(x, x^{\prime}\right)\right] d\left(x^{\prime}, x^{\prime}\right)}{1+d\left(y, y^{\prime}\right)+d\left(x, x^{\prime}\right)}\right] .
\end{aligned}
$$

Since $d(x, x)=d\left(x^{\prime}, x^{\prime}\right)=0$, we have:

$$
\begin{aligned}
d\left(x, x^{\prime}\right) & \leq\left(a_{1}+a_{3}+a_{6}\right) d\left(x, x^{\prime}\right)+\left(a_{3}+a_{6}\right) d\left(x^{\prime}, x\right)+a_{1} d\left(y, y^{\prime}\right) \\
\left(1-\left(a_{1}+a_{3}+a_{6}\right)\right) d\left(x, x^{\prime}\right) & \leq\left(a_{3}+a_{6}\right) d\left(x^{\prime}, x\right)+a_{1} d\left(y, y^{\prime}\right) .
\end{aligned}
$$

By following the similar procedure, we can get:

$$
\left(1-\left(a_{1}+a_{3}+a_{6}\right)\right) d\left(y, y^{\prime}\right) \leq\left(a_{3}+a_{6}\right) d\left(y^{\prime}, y\right)+a_{1} d\left(x, x^{\prime}\right) .
$$

Adding (8) and (9) and then simplifying, we obtain:

$$
\left[d\left(x, x^{\prime}\right)+d\left(y, y^{\prime}\right)\right] \leq \sigma\left[d\left(x^{\prime}, x\right)+d\left(y^{\prime}, y\right)\right] .
$$

where

$$
\sigma=\left[\frac{a_{3}+a_{6}}{1-\left(2 a_{1}+a_{3}+a_{6}\right)}\right] .
$$

Similarly, we can get:

$$
\left[d\left(x^{\prime}, x\right)+d\left(y^{\prime}, y\right)\right] \leq \sigma\left[d\left(x, x^{\prime}\right)+d\left(y, y^{\prime}\right)\right]
$$

Adding (10) and (11), we get:

$$
\left[d\left(x, x^{\prime}\right)+d\left(y, y^{\prime}\right)+d\left(x^{\prime}, x\right)+d\left(y^{\prime}, y\right)\right] \leq \sigma\left[d\left(x, x^{\prime}\right)+d\left(y, y^{\prime}\right)+d\left(x^{\prime}, x\right)+d\left(y^{\prime}, y\right)\right] .
$$

Since $\sigma<1$ so, the above inequality is possible if and only if $\left[d\left(x, x^{\prime}\right)+d\left(y, y^{\prime}\right)+d\left(x^{\prime}, x\right)+d\left(y^{\prime}, y\right)\right]=0$. Which implies that $d\left(x, x^{\prime}\right)=0, d\left(y, y^{\prime}\right)=0, d\left(x^{\prime}, x\right)=0$, and $d\left(y^{\prime}, y\right)=0$. It follows that $x=x^{\prime}$ and $y=y^{\prime}$ such that $(x, y)=\left(x^{\prime}, y^{\prime}\right)$ which contradicts our assumption. Therefore, $(x, y)$ is a unique coupled fixed point of $T$ in $X \times X$.

Remark 1. If we take $k=1$ and $a_{7}=0$ in Theorem 3, we get Theorem 2 of [8]. Thus our established theorem generalizes Theorem 2 .

Example 1. Let $X=[0,1]$. Define $d: X \times X \rightarrow \Re^{+}$by

$$
d(x, y)=|2 x+y|^{2}+|2 x-y|^{2}
$$

for all $x, y \in X$. Then $(X, d)$ is dq b-metric space with constant coefficient $k=2$. If we define a continuous map $T: X \times X \rightarrow X$ by $T(x, y)=\frac{x y}{10}$. Since $|2 x y+u v|^{2} \leq|2 x+u|^{2}+|2 y+v|^{2},|2 x y-u v|^{2}<|2 x-u|^{2}+|2 y-v|^{2}$ holds for all $x, y, u, v \in X$. Then, we have:

$d[T(x, y), T(u, v)]=d\left(\frac{2 x y}{10}, \frac{u v}{10}\right)=\left|\frac{2 x y}{10}+\frac{u v}{10}\right|^{2}+\left|\frac{2 x y}{10}-\frac{u v}{10}\right|^{2} \leq \frac{1}{100}\left(|2 x+u|^{2}+|2 y+v|^{2}+|2 x-u|^{2}+|2 y-v|^{2}\right)$ $=\frac{1}{10}[d(x, u)+d(y, v)]$. 
It shows that:

$$
\begin{aligned}
d[T(x, y), T(u, v)] \leq & a_{1}[d(x, u)+d(y, v)]+a_{2}[d(x, T(x, y))+d(u, T(u, v))]+a_{3}[d(x, T(u, v))+d(u, T(x, y))] \\
& +a_{4}\left[\frac{d(x, T(x, y)) d(u, T(u, v))}{d(x, u)+d(y, v)}\right]+a_{5}\left[\frac{[d(x, u)+d(y, v)][d(x, T(x, y))+d(u, T(u, v))]}{1+d(x, u)+d(y, v)}\right] \\
& +a_{6}\left[\frac{d(u, T(x, y))+d(x, T(u, v))}{1+d(u, T(u, v)) d(u, T(x, y))}\right]+a_{7}\left[\frac{[d(y, v)+d(x, T(x, y))] d(u, T(u, v))}{1+d(y, v)+d(x, T(x, y))}\right] .
\end{aligned}
$$

It can be easily shown that $(0,0) \in X \times X$ is the unique coupled fixed point of $T$ in $X \times X$. Hence, we have successfully verified Theorem 3.

\section{Conclusion and future scope}

Mohammed et al. [8] proved the existence and uniqueness of a coupled fixed point result for maps satisfying certain rational type contractive condition in the setting of complete dislocated quasi metric space. In this paper, we have established and proved the existence and uniqueness of a coupled fixed point result for maps satisfying rational type contractive condition in the perspective of complete dislocated quasi b- metric spaces. Our established result generalizes and extends the result of Mohammed et al. [8] and related results in the existing literature. Also, we provided examples in support of the main result. The researchers believes that the search for the existence and uniqueness of coupled fixed point for maps satisfying different contractive conditions in dislocated quasi b- metric space is an active area of research. As a result, any interested researchers can utilize this opportunity to conduct their thesis work in this area.

Acknowledgments: The authors would like to thank the College of Natural Sciences, Jimma University for funding this research work.

Author Contributions: All authors contributed equally to the writing of this paper. All authors read and approved the final manuscript.

Conflicts of Interest: "The authors declare no conflict of interest."

\section{References}

[1] Hitzler, P., \& Seda, A. K. (2000). Dislocated topologies. Journal of electrical engineering, 51(12), 3-7.

[2] Hitzler, P. (2001). Generalized metrics and topology in logic programming semantics [Ph. D. thesis]. National University of Ireland (University College, Cork).

[3] Zeyada, F. M., Hassan, G. H., \& Ahmed, M. A. (2006). A generalization of a fixed point theorem due to Hitzler and Seda in dislocated quasi-metric spaces. Arabian journal for Science and Engineering, 31(1A), 111-114.

[4] Rahman, M. U., \& Sarwar, M. (2016). Dislocated quasi b-metric space and fixed point theorems. Electronic Journal of Mathematical Analysis and Applications, 4(2), 16-24.

[5] Guo, D., \& Lakshmikantham, V. (1987). Coupled fixed points of nonlinear operators with applications. Nonlinear Analysis: Theory, Methods \& Applications, 11(5), 623-632.

[6] Bhaskar, T. G., \& Lakshmikantham, V. (2006). Fixed point theorems in partially ordered metric spaces and applications. Nonlinear Analysis: Theory, Methods \& Applications, 65(7), 1379-1393.

[7] Al Muhiameed, Z., Bousselsal, M., \& Mostefaoui, Z. (2018). Some Coupled Fixed Point Results in Discolated Quasi-b-Metric Spaces for Rational Type Contraction Mappings. Asian Journal of Mathematics and Physics, 2, 1-5.

[8] Mohammad, M., Jamal, R., Mishra, J., Kabir, Q. A., \& Bhardwaj, R. (2018). Coupled Fixed Point Theorem and Dislocated Quasi-Metric Space. International Journal of Pure and Applied Mathematics, 119(10), 1249-1260.

[9] Banach, S. (1922). Sur les opérations dans les ensembles abstraits et leur application aux équations intégrales. Fundamenta Mathematicae 3(1), 133-181.

[10] Rahman, M. U. (2017). New fixed point theorems in dislocated quasi- b-metric space. Applied Mathematics $\mathcal{E}$ Information Sciences Letters, 5 (1), 7-11. 